\title{
Different sonographic features of peripheral thyroid nodule calcification and risk of malignancy: a prospective observational study
}

\author{
Mehrdad Nabahati ${ }^{1 A, B, D, E}$, Naser Ghaemian ${ }^{1 A, D, E}$, Zoleika Moazezi ${ }^{2 B, E, F}$, Rahele Mehraeen ${ }^{3 D, E, F}$ \\ 'Department of Radiology, Shahid Beheshti Hospital, Babol University of Medical Sciences, Babol, Iran \\ 2Department of Endocrinology, Rohani Hospital, Babol University of Medical Sciences, Babol, Iran \\ ${ }^{3}$ Department of Radiology, Rohani Hospital, Babol University of Medical Sciences, Babol, Iran
}

\begin{abstract}
Purpose: To investigate the association of peripheral calcification, as well as its sonographic features, with thyroid nodule malignancy.

Material and methods: This study was prospectively conducted during 2015-2020 on patients diagnosed with thyroid nodule undergoing ultrasound-guided fine-needle aspiration in Shahid Beheshti teaching hospital or private offices in Babol, northern Iran. The ultrasonographic characteristics of the nodules, as well as the cytological findings, were recorded. Regression analysis was used to assess the relationship between sonographic results and malignancy. We also used receiver operator characteristics (ROC) analysis to estimate the ability of ultrasound to predict the characteristic features of malignancy, as estimated by the area under the curve (AUC).

Results: A total of 1857 thyroid nodules were finally included, of which 84 were peripherally calcified nodules. There was a significant positive association between the nodule malignancy and peripheral calcification $(\mathrm{OR}=2.23,95 \% \mathrm{CI}$ : 1.13-4.35). In the nodules with peripheral calcification, significant positive associations were seen between malignancy and lobulated margin ( $\mathrm{OR}=3.85,95 \% \mathrm{CI}$ : 1.02-14.54) and solid composition (OR $=4.05,95 \% \mathrm{CI}$ : 0.99-16.53). The ROC analysis indicated that AUC for lobulated margin and solid composition was $63.8 \%$ and $66.5 \%$, respectively, in predicting malignant thyroid nodules.

Conclusion: The findings showed that peripheral calcification on sonography can be a potential indicator of malignant thyroid nodules. Also, the presence of lobulated margin and/or solid composition, besides peripheral calcification, can be helpful in better distinguishing malignant from benign nodules.
\end{abstract}

Key words: thyroid nodule, ultrasonography, peripheral calcification, fine-needle aspiration.

\section{Introduction}

Thyroid nodules are abnormal overgrowths of tissue-forming masses in the thyroid gland. They are common and can be observed in up to $68 \%$ in adults [1]. It has also been stated that $5-15 \%$ of the thyroid nodules are malignant [2]; therefore, the possibility of cancer should be considered for every nodule. In recent decades, advanced diagnostic techniques, like ultrasonography and fine-needle aspiration
(FNA), have been widely used. Nevertheless, the conventional and practical approaches to investigate and diagnose thyroid nodules have been controversial $[3,4]$.

One of the sonographic criteria for differentiating benign and malignant thyroid nodules is calcification or echogenic foci, which can be observed in 14-55\% of the thyroid nodules, associated with 29-59\% malignancy rate [5]. Based on the American College of Radiology (ACR) Thyroid Imaging Reporting and Data System (TI-RADS), 3 types of

Correspondence address:

Naser Ghaemian, Department of Radiology, Shahid Beheshti Hospital, Babol University of Medical Sciences, Babol, Iran, e-mail: nghaemian@gmail.com

Authors' contribution:

A Study design · B Data collection · C Statistical analysis · D Data interpretation · E Manuscript preparation · F Literature search · G Funds collection 
echogenic foci are potentially linked to malignancy [6,7]: macrocalcifications, punctate echogenic foci, and peripheral rim calcifications (or eggshell calcifications). Macrocalcifications have been reported to carry a mild risk of malignancy, while punctate echogenic foci, which are mostly accompanied by psammomatous calcifications in papillary thyroid cancer, have been regarded with a high possibility of malignancy $[8,9]$. Nevertheless, the literature on the malignancy probability of rim calcifications has remained controversial $[10,11]$. In the present study, we investigated the risk of cancer in thyroid nodules with peripheral calcification. Furthermore, we tried to determine the sonographic features of peripheral calcification, which are probably associated with increased thyroid nodule malignancy.

In many areas of Iran, thyroid nodules are frequently seen because of dietary iodine deficiency. Thus, an accurate diagnostic approach, which can exclude the malignancy risk, is necessary to ascertain the nature of the thyroid nodule. In this study, we aimed to specifically focus on the diagnostic accuracy of peripheral thyroid nodule calcification as well as its different sonographic features. Considering that there are insufficient data on the diagnostic accuracy of peripheral calcification to differentiate malignant from benign nodules, especially in our region, there was a need to perform the present study.

\section{Material and methods}

\section{Locations and patients}

In this prospective cross-sectional study, patients with thyroid nodules consecutively undergoing ultrasoundguided FNA in the clinics of Shahid Beheshti teaching hospital or private offices in Babol, northern Iran, from March 2015 to February 2020, were recruited. Subjects with purely cystic nodules with no solid focus, those who were unwilling to conduct FNA, and cases with suspected cytology findings (atypia) were excluded. Specifically, in the present study, the subjects with peripherally calcified nodules were finally considered for additional analyses.

\section{Ultrasound imaging and fine-needle aspiration}

The sonographic criteria for considering a nodule as suspicious for malignancy included hypoechogenicity, calcification, lobulated margins, and/or a taller-than-wide shape. Peripheral calcification was defined as calcification that lies along all (continuous) or part of (interrupted) the margin of a nodule [6]. An expert radiologist performed the ultrasound-guided FNA on the nodules using a Samsung H60 ultrasound machine (a 3-14 MHz linear array transducer) and a 23-gauge needle attached to a $5 \mathrm{cc}$ syringe, using a freehand technique. Regarding the solidcystic nodules, FNA was done from the solid areas of the samples.

\section{Fine-needle aspiration cytology}

We transferred the samples to the laboratory on smear glass slides after drying in the open air and fixing with $95 \%$ alcohol. To stain the slides, the Papanicolaou, Giemsa, and haematoxylin and eosin methods were used. To control the bias, the cytohistological examinations of all samples were performed by a single expert pathologist, who was also blinded to the ultrasound diagnosis of thyroid nodules. Some samples were examined by 2 pathologists when decision making was difficult.

\section{Data collection}

The following data were collected from the patients undergoing ultrasound-guided FNA:

- demographic information, including age and sex;

- ultrasonographic characteristics of the thyroid nodules, including the size of the nodule $(<2 \mathrm{~cm}$ or $>2 \mathrm{~cm})$, nodule echogenicity (hyper, hypo, iso), peripheral calcification continuity (continuous, interrupted), margins of the nodule (regular, lobulated), and nodule composition (solid, solid-cystic);

- cytological results.

The data were recorded in a checklist form. Surgery was performed for the malignant thyroid nodules.

\section{Statistical analysis}

SPSS software was used for data analysis. To summarize the data, descriptive statistics were used. To statistically measure the diagnostic accuracy of microcalcification features, the contingency table values were defined as follows:

- true positive (TP): thyroid nodule was determined to be malignant in both ultrasound and cytology;

- true negative (TN): thyroid nodule was established as benign in both ultrasound and cytology;

- false positive (FP): ultrasonography was suggestive of malignancy, but cytopathology was inconsistent;

- false negative (FN): ultrasonography did not show malignancy, but cytology suggested it.

Sensitivity was calculated as $\mathrm{TP} / \mathrm{TP}+\mathrm{FN}$, specificity as $\mathrm{TN} / \mathrm{TN}+\mathrm{FP}$, positive predictive value (PPV) as TP/ $\mathrm{TP}+\mathrm{FP}$, negative predictive value (NPV) as TN/TN+FN, and accuracy as proportion of $\mathrm{TP}+\mathrm{TN}$ in all patients. The association between ultrasonographic features and malignancy was assessed using logistic regression analysis. The data were presented as odds ratio (OR) as well as 95\% confidence interval (CI). A receiver operator characteristics (ROC) analysis was also used to estimate the ability of sonography to predict characteristic features of malignancy, as estimated by the area under the curve (AUC). A $p$-value less than 0.05 was considered as significant. 


\section{Ethical issues}

The details of this study were initially explained to the patients, and then written informed consent was obtained from each of them. The study protocol was approved by the ethics committee of Babol University of Medical Sciences (code: IR.MUBABOL.REC.1399.223). The patients' information was kept confidential.

\section{Results}

Overall, 1793 patients with thyroid nodules underwent ultrasound-guided FNA. Of these, 1545 had single nodules and the others had multiple nodules. In total, 1985 thyroid nodules were initially assessed, of which 128 were atypia according to cytology and were excluded from further investigation. A total of 1857 nodules (from 1641 patients) were finally included in the study, of which 84 were peripherally calcified nodules. The number of female patients was 1293 (83.9\%), and others were male. The mean age of the patients was $44.8 \pm 13.9$ years. The mean size of the nodules was $1.97 \pm 1.22 \mathrm{~cm}$. The number of benign and malignant nodules was 1674 (90.1\%) and 183 (9.9\%), respectively.
Peripheral calcification was observed in $6 \%(n=11)$ of malignant nodules. On the other hand, malignancy was seen in $13.1 \%(n=11)$ of nodules with peripheral calcification, compared with $6.4 \%(n=82)$ found in nodules with no calcification. In this regard, there was a significant positive association between nodule malignancy and peripheral calcification $(\mathrm{OR}=2.23,95 \% \mathrm{CI}$ : 1.13-4.35; $p=0.02)$. The ROC analysis showed that AUC for peripheral calcification was $50.8 \%$ in predicting malignant thyroid nodules. The calculated sensitivity, specificity, PPV, NPV, and accuracy for peripheral calcification were $6 \%, 95.6 \%, 13.1 \%, 90.3 \%$, and $86.8 \%$, respectively.

Out of 84 nodules with peripheral calcification, 73 $(86.9 \%)$ were benign and $11(13.1 \%)$ were malignant. The cytology findings proved that $84.5 \%$ of the nodules were nodular goitre, $13.1 \%$ were papillary thyroid carcinoma, and $2.4 \%$ were thyroiditis. Table 1 shows the association between the cytology results (benign or malignant) and the sonographic features of the thyroid nodules with peripheral calcification. There were significant positive associations between malignancy and lobulated margin $(\mathrm{OR}=3.85$, 95\% CI: $1.02-14.54)$ and solid composition (OR $=4.05$, 95\% CI: 0.99-16.53). The mean size between the benign and malignant nodules was $1.65 \pm 0.93$ and $1.51 \pm 0.45 \mathrm{~cm}$,

Table 1. Association between sonographic characteristics and cytology results of the peripherally calcified thyroid nodules

\begin{tabular}{|c|c|c|c|c|}
\hline Sonographic features & Benign, $n(\%)$ & Malignant, $n(\%)$ & $p$-value & Odds ratio $(95 \% \mathrm{Cl})$ \\
\hline \multicolumn{5}{|l|}{ Nodule size $(\mathrm{cm})$} \\
\hline$<2$ & $50(84.7)$ & $9(15.3)$ & \multirow[t]{2}{*}{0.37} & 1 \\
\hline$\geq 2$ & $23(92.0)$ & $2(8.0)$ & & $0.48(0.10-2.42)$ \\
\hline \multicolumn{5}{|l|}{ Margin } \\
\hline Regular & $60(90.9)$ & $6(9.1)$ & \multirow[t]{2}{*}{0.04} & 1 \\
\hline Lobulated & $13(72.2)$ & $5(27.8)$ & & $3.85(1.02-14.54)$ \\
\hline \multicolumn{5}{|l|}{ Echogenicity } \\
\hline Hyperechogenicity & $28(90.3)$ & $3(9.7)$ & & 1 \\
\hline Isoechogenicity & $33(91.7)$ & $3(8.3)$ & 0.85 & $0.85(0.16-4.54)$ \\
\hline Hypoechogenicity & $12(70.6)$ & $5(29.4)$ & 0.09 & $3.89(0.80-18.94)$ \\
\hline \multicolumn{5}{|l|}{ Composition } \\
\hline Solid-cystic & $44(93.6)$ & $3(6.4)$ & \multirow[t]{2}{*}{0.04} & 1 \\
\hline Solid & $29(78.4)$ & $8(21.6)$ & & $4.05(0.99-16.53)$ \\
\hline \multicolumn{5}{|l|}{ Calcification continuity } \\
\hline Interrupted & $38(88.4)$ & $5(11.6)$ & \multirow[t]{2}{*}{0.68} & 1 \\
\hline Continuous & $35(85.4)$ & $6(14.6)$ & & $1.30(0.37-4.65)$ \\
\hline
\end{tabular}

Table 2. Diagnostic value of sonographic features in predicting malignancy for the peripherally calcified thyroid nodules

\begin{tabular}{|l|c|c|c|c|c|}
\hline $\begin{array}{l}\text { Ultrasound } \\
\text { features }\end{array}$ & $\begin{array}{c}\text { Sensitivity } \\
(\%, 95 \% \mathrm{Cl})\end{array}$ & $\begin{array}{c}\text { Specificity } \\
(\%, 95 \% \mathrm{Cl})\end{array}$ & $\begin{array}{c}\text { Positive predictive } \\
\text { value }(\%, 95 \% \mathrm{Cl})\end{array}$ & $\begin{array}{c}\text { Negative predictive } \\
\text { value }(\%, 95 \% \mathrm{Cl})\end{array}$ & $\begin{array}{c}\text { Accuracy } \\
(\%, 95 \% \mathrm{Cl})\end{array}$ \\
\hline Lobulated margin & $45.5(16.8-76.6)$ & $82.2(71.5-90.2)$ & $27.8(14.6-46.5)$ & $90.9(85.2-94.5)$ & $77.4(67-84.8)$ \\
\hline Solid composition & $21.6(9.8-38.2)$ & $93.6(82.5-98.7)$ & $72.7(43.2-90.3)$ & $60.3(55.8-64.6)$ & $61.9(50.7-72.3)$ \\
\hline
\end{tabular}




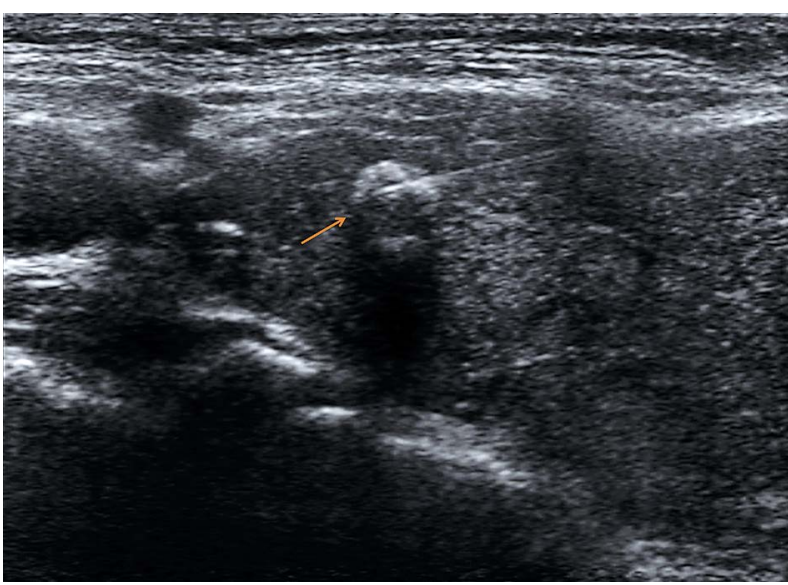

Figure 1. Ultrasound-guided fine-needle aspiration from an isoechoic nodule with interrupted peripheral calcification with $8 \mathrm{~mm}$ diameter in the right thyroid lobe of a 39 -year-old female patient, which was proven by cytology to be a nodular goitre. The procedure was done following the patient's request due to history of papillary thyroid carcinoma in her sister

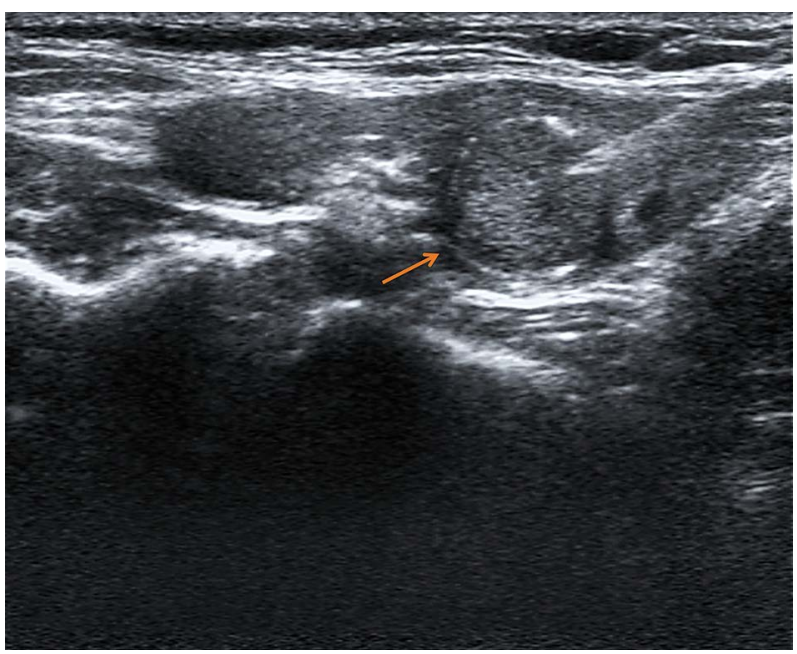

Figure 2. Ultrasound-guided fine-needle aspiration from an isoechoic solid nodule with peripheral calcification in the right thyroid lobe of a 41-yearold female patient, which was proven by cytology to be a papillary thyroid carcinoma

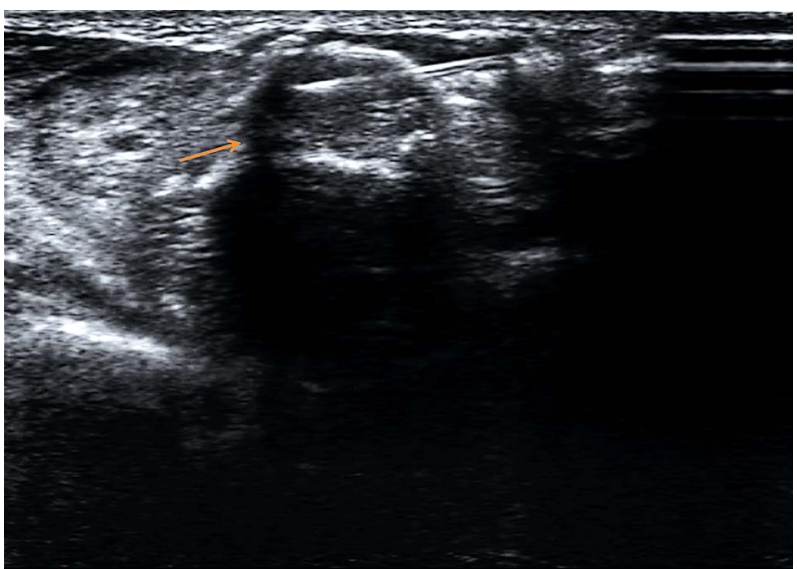

Figure 3. Ultrasound-guided fine-needle aspiration from a hypoechoic solid nodule with peripheral calcification in the right thyroid lobe of a 46-yearold female patient, which was proven by cytology to be a nodular goitre respectively $(p=0.63)$. The ROC analysis indicated that AUC for lobulated margin and solid composition was $63.8 \%$ and $66.5 \%$, respectively, in predicting malignant thyroid nodules. The computed sensitivity, specificity, PPV, NPV, and accuracy for these sonographic features have also been demonstrated in Table 2. Figures 1-3 also show the ultrasound-guided FNA of benign and malignant nodules.

\section{Discussion}

According to our results, peripheral calcification was associated with increased risk of thyroid nodule malignancy. There are conflicting reports about the relation between macrocalcifications and thyroid nodule malignancy; however, most of the results are in favour of a direct association between peripheral calcification and nodule malignancy [12-15], and our results supports such a theory. We also calculated the sensitivity for the peripheral calcification to be $6 \%$. In contrast, the specificity was as high as $95.6 \%$. The low sensitivity was not unexpected and could be explained by the fact that peripheral calcification is generally observed at a low rate on thyroid nodule sonography. One of the reasons can be related to the different challenges for the nodule biopsy, such as difficulty in penetrating the shell and seeing the needle tip within the lesion because of obscuring shadowing.

In the present study, it was found that lobulated margin could be predictive for malignancy in the nodules with peripheral calcification, with high specificity but lower sensitivity. Previous surveys also revealed that irregular margin or microlobulated margin are potential predictors of thyroid cancer, but a regular margin is linked to both benign and malignant lesions [2,16,17]. Also, according to the ACR TI-RADS guidelines, rim calcification accompanied by a lobulated margin is associated with a higher score than rim calcification alone [7]. Altogether, number of studies evaluating these relations in peripherally calcified nodules is limited and more surveys are needed.

Solid structure was another sonographic feature that was significantly associated with a higher thyroid malignancy risk in the peripherally calcified nodules, which was congruent with the previous surveys $[17,18]$, as well as ACR TI-RADS guidelines, in which a combination of rim calcification with solid composition has a higher score than rim calcification alone [7]. On the other hand, researchers generally consider the cystic lesions as benign, unless a solid component is present. A recent study by $\mathrm{Na}$ et al. [19] showed that thyroid nodules with minimal cystic changes (defined as presence of an anechoic cystic portion within a nodule), as well as partially cystic nodules (cystic portion $>10 \%$ ), have a low malignancy risk. Altogether, it can be stated that in the thyroid nodules with peripheral calcification, simultaneous presence of a cystic component and solid structure, compared with solid composition alone, will probably decrease the diagnostic value for predicting malignancy. 
According to the findings, the size of the thyroid nodules was not identified as a potential predictor of malignancy in the peripherally calcified nodules. There are conflicting findings on the impact of size on thyroid cancer risk, and both positive and negative associations have been reported between size and malignancy risk [20]. In their study, Kamran et al. [21] detected a threshold at $2 \mathrm{~cm}$, and nodules with higher sizes were directly correlated with increased risk of malignancy.

In our study, no association was seen between echogenicity and thyroid nodule malignancy risk in the nodules with peripheral calcification. It has been reported that malignancy is observed in $4 \%$ of solid hyperechoic nodules, but this rate rises to $26 \%$ for hypoechoic nodules [22]. Despite some conflicting consequences, the majority of papers alluded to an inverse association between echogenicity degree and thyroid cancer risk $[23,24]$. A recent meta-analysis also reported that the estimated sensitivity for hypoechogenicity in unselected nodules was 62.7\% [17].

Our analyses revealed no significant value for the peripheral calcification continuity in distinguishing benign from malignant lesions. There is controversy about this subject. A recent study by Malhi et al. [25] indicated similar findings to ours, and difficulty of accurately assessing this feature was mentioned as a cause of these non-significant results. On the other hand, Yin et al. [26] stated that eggshell discontinuous calcifications and multilayer-like calcifications were directly related to thyroid carcinoma. Altogether, more studies are needed to clarify these associations.

A limitation of the present study was inaccessibility of repeat FNA findings in some cases with the atypia. In addition, we did not have the pathological findings of malignant thyroids of patients who underwent surgery. Therefore, we suggest that new studies are designed to compare the sonographic and FNA results with pathological results. Moreover, multicentre studies with lager sample sizes are recommended to enable more generalizable outcomes.

A strength of the present study is its prospective design compared to previous studies, which were retrospectively performed. Therefore, our results are potentially more precise compared with other studies due to various issues in data collection (e.g. more accurate data recording and less recall bias). Another strength is the relatively large number of thyroid nodules assessed in this study.

\section{Conclusions}

The findings of the present study show that peripheral calcification on sonography can be a potential indicator of malignant thyroid nodules. Also, the presence of lobulated margin and solid composition, in addition to peripheral calcification, can improve the ability of peripheral calcification in distinguishing malignant from benign nodules. Continuity of peripheral calcification is a notable subject, and its association with thyroid cancer risk should be clarified by further studies.

\section{Acknowledgements}

We would like to thank the Vice Chancellor for the Research of Babol University of Medical Sciences for financial support. We are also thankful to Dr. Sharbatdaran for his help in cytological assessments.

\section{Conflict of interest}

The authors report no conflict of interest.

\section{References}

1. Karkada M, Costa AF, Imran SA, et al. Incomplete thyroid ultrasound reports for patients with thyroid nodules: implications regarding risk assessment and management. Am Roentgen Ray Soc 2018; 211: 1348-1353.

2. Shen Y, Liu M, He J, et al. Comparison of different risk-stratification systems for the diagnosis of benign and malignant thyroid nodules. Front Oncol 2019; 9: 378.

3. Tamhane S, Gharib H. Thyroid nodule update on diagnosis and management. Clin Diabetes Endocrinol 2016; 2: 17.

4. McQueen AS, Bhatia KS. Thyroid nodule ultrasound: technical advances and future horizons. Insights Imaging 2015; 6: 173 188.

5. Ha SM, Chung YJ, Ahn HS, et al. Echogenic foci in thyroid nodules: diagnostic performance with combination of TIRADS and echogenic foci. BMC Med Imaging 2019; 19: 28.

6. Jabar ASS, Koteshwara P, Andrade J. Diagnostic reliability of the Thyroid Imaging Reporting and Data System (TI-RADS) in routine practice. Pol J Radiol 2019; 84: e274-e280.
7. Tessler FN, Middleton WD, Grant EG, et al. ACR thyroid imaging, reporting and data system (TI-RADS): white paper of the ACR TI-RADS committee. J Am Coll Radiol 2017; 14: 587-595.

8. Erdem Toslak I, Martin B, Barkan GA, et al. Patterns of sonographically detectable echogenic foci in pediatric thyroid carcinoma with corresponding histopathology: an observational study. AJNR Am J Neuroradiol 2018; 39: 156-161.

9. Gwon HY, Na DG, Noh BJ, et al. Thyroid nodules with isolated macrocalcifications: malignancy risk of isolated macrocalcifications and postoperative risk stratification of malignant tumors manifesting as isolated macrocalcifications. Korean J Radiol 2020; 21: 605-613.

10. Shin JH, Baek JH, Chung J, et al. Ultrasonography diagnosis and imaging-based management of thyroid nodules: revised Korean Society of Thyroid Radiology consensus statement and recommendations. Korean J Radiol 2016; 17: 370-395.

11. Kim BM, Kim MJ, Kim EK, et al. Sonographic differentiation of thyroid nodules with eggshell calcifications. J Ultrasound Med 2008; 27: 1425-1430. 
12. Park YJ, Kim JA, Son EJ, et al. Thyroid nodules with macrocalcification: sonographic findings predictive of malignancy. Yonsei Med J 2014; 55: 339-344.

13. Xie C, Cox P, Taylor N, LaPorte S. Ultrasonography of thyroid nodules: a pictorial review. Insights Imaging 2016; 7: 77-86.

14. Lee YH, Kim DW, In HS, et al. Differentiation between benign and malignant solid thyroid nodules using an US classification system. Korean J Radiol 2011; 12: 559-567.

15. Nabahati M, Moazezi Z, Fartookzadeh S, et al. The comparison of accuracy of ultrasonographic features versus ultrasound-guided fine-needle aspiration cytology in diagnosis of malignant thyroid nodules. J Ultrasound 2019; 22: 315-321.

16. Siebert SM, Gomez AJ, Liang T, et al. Diagnostic performance of margin features in thyroid nodules in prediction of malignancy. AJR Am J Roentgenol 2018; 210: 860-865.

17. Remonti LR, Kramer CK, Leitao CB, et al. Thyroid ultrasound features and risk of carcinoma: a systematic review and meta-analysis of observational studies. Thyroid 2015; 25: 538-550.

18. Popoveniuc G, Jonklaas J. Thyroid nodules. Med Clin North Am 2012; 96: 329-349.

19. Na DG, Kim JH, Kim DS, Kim SJ. Thyroid nodules with minimal cystic changes have a low risk of malignancy. Ultrasonography 2016; 35: 153-158

20. Al-Hakami HA, Alqahtani R, Alahmadi A, et al. Thyroid nodule size and prediction of cancer: a study at tertiary care hospital in Saudi Arabia. Cureus 2020; 12: e7478.

21. Kamran SC, Marqusee E, Kim MI, et al. Thyroid nodule size and prediction of cancer. J Clin Endocrinol Metab 2013; 98: 564-570.

22. Anil G, Hegde A, Chong FV. Thyroid nodules: risk stratification for malignancy with ultrasound and guided biopsy. Cancer Imaging 2011; 11: 209-223.

23. Lee JY, Na DG, Yoon SJ, et al. Ultrasound malignancy risk stratification of thyroid nodules based on the degree of hypoechogenicity and echotexture. Eur Radiol 2020; 30: 1653-1663.

24. Tugendsam C, Petz V, Buchinger W, et al. Ultrasound criteria for risk stratification of thyroid nodules in the previously iodine deficient area of Austria - a single centre, retrospective analysis. Thyroid Res 2018; 11: 3.

25. Malhi HS, Velez E, Kazmierski B, et al. Peripheral thyroid nodule calcifications on sonography: evaluation of malignant potential. AJR Am J Roentgenol 2019; 213: 672-675.

26. Yin L, Zhang W, Bai W, He W. Relationship between morphologic characteristics of ultrasonic calcification in thyroid nodules and thyroid carcinoma. Ultrasound Med Biol 2020; 46: 20-25. 